\title{
Preparing old and recent radio source tables for the VO age: Current status
}

\author{
Heinz Andernach*a,b \\ ${ }^{a}$ Argelander-Institut für Astronomie (AIfA), Universität Bonn, Auf dem Hügel 71 \\ D-53121 Bonn, Germany \\ ${ }^{b}$ On leave of absence from Depto. de Astronomía, Universidad de Guanajuato, AP 144 \\ Guanajuato, CP 36000, Gto, Mexico \\ E-mail: heinz@astro.uni-bonn.de
}

Independent of established data centers, and partly for my own research, I have been collecting the tabular data from nearly 1500 articles concerned with radio sources. Optical character recognition (OCR) was used to recover tables from nearly 600 of these. Tables from only 44 percent of these articles are available in the CDS or CATS catalog collections. This fraction is 62 percent for articles with over 100 sources. Surprisingly, these fractions are not better for articles published electronically since 2001, perhaps partly due to the fact that often tabular data are published in formats not useful for direct machine reading. The databases Simbad and NED recognize only about 60 percent of the bibliographic references corresponding to the existing electronic radio source lists, and the number of objects associated with these references is much smaller still. Both, object databases like NED and Simbad, as well as catalog browsers (VizieR, CATS) need to be consulted to obtain the most complete information on radio sources. More human resources at the data centers and better collaboration between authors, referees, editors, publishers, and data centers are required to improve the flow of tabular data from journals to public databases. Current efforts within the Virtual Observatory (VO) project, to provide retrieval and analysis tools for different types of published and archival data stored at various sites, should be balanced by an equal effort to recover and include large amounts of published data not currently available in this way. If human resources can be found, the data sets collected by the author will be made available for the preparation of metadata necessary for their ingression into catalog browsers.

Panoramic Radio Astronomy: Wide-field 1-2 GHz research on galaxy evolution

June 2-5 2009

Groningen, the Netherlands

${ }^{*}$ Speaker. 


\section{Introduction}

Since 1989, motivated by a lack of data on radio sources in NED (nedwww.ipac.caltech.edu), Simbad (simbad.u-strasbg.fr), and the CDS catalogs (cdsarc.u-strasbg.fr/viz-bin/ftp-index), I collected electronic tables of radio sources and/or extragalactic objects that (a) contained $\gtrsim 50$ records, and (b) were unavailable from data centers at the time. Many of these catalogs were later incorporated in the CDS archive and the VizieR catalog browser (vizier.u-strasbg.fr, [2]), as well as in the CATS catalog browser (cats.sao.ru, [3]). My collection of data tables from currently 2950 articles is described in more detail (as of Sep. 2008) in [1]. Here I concentrate on the subset of tables from 1494 articles dealing with radio sources. Tables from about 580 of these, published 1950-1999 and with a total of $\sim 200,000$ records, were recovered with optical character recognition (OCR) software. Since 2004 the collection has grown by $\sim 110$ articles/yr, half of these published before 1999 and recovered via OCR, sometimes correcting the raw OCR results of the Astrophysics Data System (adsabs.harvard.edu/cgi-bin/signup_ocr). Tables from a further $\sim 110$ articles at CDS/VizieR were not duplicated into my collection. Thus, the total number of articles with existing electronic radio source tables is currently $\sim 1600$. The cumulative size distribution of the latter is shown by the continuous line in Figure 1. Here, size is typically the number of objects in a catalog, but may also be the number of flux density measurements, e.g. in case of various observing frequencies or epochs. The size distribution closely follows a power law with a slope of -0.70 . Such power laws are known in biometrics as Zipf's or Lotka's laws. For sizes $\lesssim 100$ records a "collection bias" sets in, caused by working from the bigger towards the smaller lists.

\section{Comparison with other catalog browsers}

The largest collection of astronomical catalogs is maintained by CDS at Strasbourg, where, as of Sept. 2009, 8000 catalogs are accessible for download, and 7560 are also available for cone searches through the VizieR browser. The dashed line in Fig. 1 indicates the size distribution of radio catalogs in the CDS collection, which is complete for radio catalogs of $\gtrsim 10,000$ records. The dotted line shows the same for CATS, which is complete for $\gtrsim 5000$ records.

The left side of Table 1 lists the number of items in each collection of radio catalogs, followed by the percentage in number of catalogs, their total number of records, and their percentage in number of records, as of 1-Sep-2009. HA stands for the author, and the third row refers to catalogs which are in the author's, but not in the CDS or CATS collections. I am not aware of any electronic radio catalog outside the HA+CDS collection. For those electronic radio source catalogs with more than 100 records, only $52 \%$ are in the CDS archive, and only $34 \%$ are included in CATS. While CATS offers more data (records), VizieR offers a larger variety of catalogs, i.e. more of the smaller source lists. One needs to search in both services to obtain the most complete results, and then weed out the duplications from the 244 catalogs in common between CATS and VizieR. More than half of the items I collected are neither in VizieR nor in CATS. However, the preparation of metadata is required for their inclusion in catalog browsers. Some of these catalogs require a further effort (e.g. insert missing absolute coordinates from their object names), and a still smaller fraction of these catalogs, e.g. literature compilations or those containing only derived parameters, may be unsuitable for inclusion into catalog browsers. 


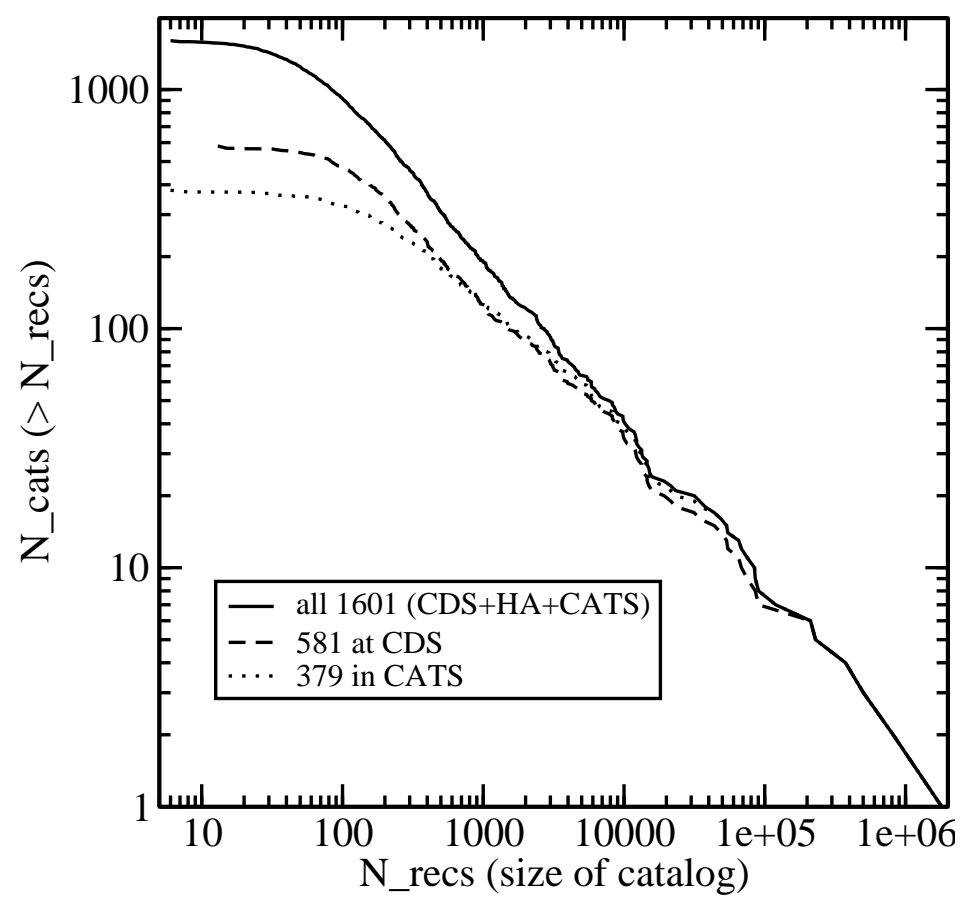

Figure 1: Cumulative size distribution of 1601 radio catalogs. For any given size (in records) listed on the abscissa, the ordinate gives the total number of catalogs up to that size (as of 1-Sep-2009). Continuous line: author's collection plus those at CDS and CATS; dashed line: subset of radio catalogs that are available at CDS; dotted line: subset of radio catalogs that are available in CATS.

\begin{tabular}{lrrrr|rrrr}
\hline & \multicolumn{3}{c}{ All radio source tables } & \multicolumn{4}{c}{ Tables with >50 records publ. since 2001 } \\
\hline Collection & Ncats & \%cats & Nrecords & \%recs & Ncats & \%cats & Nrecords & \%recs \\
\hline VizieR (not INPREP) & 581 & 36.3 & $5,269,531$ & 90.6 & 237 & 65.7 & $1,634,877$ & 94.1 \\
CATS collection & 379 & 23.7 & $5,449,164$ & 95.0 & 57 & 15.7 & $1,530,200$ & 88.1 \\
HA collection ONLY & 901 & 56.3 & 209,332 & 3.6 & 103 & 28.5 & 81,000 & 4.7 \\
HA+CDS collections & 1601 & 100.0 & $5,736,178$ & 100.0 & 361 & 100.0 & $1,737,474$ & 100.0 \\
\hline
\end{tabular}

Table 1: Left part: Presence of all electronic radio source catalogs in various collections; right part: same for the subset of tables with $>50$ records and published since 2001.

\section{Recent Catalogs published in Electronic Journals}

One might think that the vast majority of the catalogs missing from the public catalog browsers is outdated, and that in recent years there is a continuous "flow" of electronically published source tables into the data centers and catalog browsers. However, the right-hand half of Table 1 shows that this is not the case. Of the 361 radio source tables with $>50$ records, and published since 2001 , mostly in electronic journals, only $\sim 66 \%$ (albeit with $94 \%$ of the total number of records) are in VizieR. This may be due to the fact that journal tables are often published only in LaTeX, PS or PDF format, or they are not readily machine-readable for various reasons, e.g. lack of metadata, columns not aligned, or a mixture of ASCII, HTML and LaTeX codes (cf. [1]). Despite the fact that the $32 \%$ of tables missing from VizieR and CATS "only" contain 5\% of all records, these offer flux measurements at a larger variety of frequencies and epochs than the big catalogs. 


\begin{tabular}{|c|c|c|c|c|c|c|}
\hline & \multicolumn{3}{|c|}{ All 1565 radio source tables } & \multicolumn{3}{|c|}{354 tables $>50$ records, publ. since 2001} \\
\hline Service & Ncats & \%cats & \%recs & Ncats & \%cats & \%recs \\
\hline Simbad & 874 & 55.8 & 4.0 & 209 & 59.0 & 4.8 \\
\hline NED & 984 & 62.9 & 20.2 & 194 & 54.8 & 46.0 \\
\hline
\end{tabular}

Table 2: Simbad and NED's coverage for 1565 articles with radio source data (left part) and 354 articles with $>50$ records published since 2001. Columns are: number of articles with at least one object in the service, percentage of all articles, percentage of total number of objects in the service compared to the total number of records in the catalogs.

\section{Coverage of Tabular Data in Databases}

For most astronomers the major source of information on objects are databases like Simbad or NED, whose content are different from the catalog collections and browsers described above (cf. [1]). Thus I tried to estimate the fraction of tabular data covered by these databases. For 1565 of the radio source tables (exluding theses and unpublished items) both NED and Simbad were interrogated for the number of objects linked to their 19-digit refcode, and compared with the full catalog size. This is not always reasonable, as some tables give several records per astronomical objects, while other tables may not be of relevance (e.g. Galactic objects in NED). Moreover, for some of the largest datasets (e.g. NVSS, PKSCAT90) a NED search by refcode does not work. The results are listed in Table 2 for two different samples of references. Despite the caveats, a severe underrepresentation of published data is obvious: regardless of size or publication date, no more than $\sim 60 \%$ of the refcodes are recognized, and the fraction of objects included is still smaller.

\section{Conclusions}

A vast amount of the simplest form of published data (e.g. flux measurements) now exists in electronic form, but is unavailable in public web services. About half of these are readily accessible from electronic journals, and another half was painfully collected or recovered via OCR by the author. The preparation of these source lists for the ingression in catalog browsers is beyond the possibilities of the author and requires more human resources. A list of available tables can be viewed at www.astro.ugto.mx/ heinz/cats.sum, and individual data sets are available on request.

\section{Acknowledgments}

I thank the many students and secretaries who helped to recover journal tables via OCR and proofread them. Recently I enjoyed the hospitality at AIfA Bonn, Germany, with support from Mexican CONACyT grant 81356, and from German DFG through grants RE1462/2 and TRR33.

\section{References}

[1] Andernach H.: 2009, Data Science Journal, 8, 41 (arXiv:0901.2805)

[2] Ochsenbein F., Bauer P., \& Marcout J., 2000, A\&AS 143, 23

[3] Verkhodanov, O. V., Trushkin, S. A., Andernach, H., Chernenkov, V. N., 2009, Data Science Journal, 8, 34 (arXiv:0901.3118) 